ISSN: 0212-5374

\title{
ECONOMÍA DE LA EDUCACIÓN: UNA DISCIPLINA PEDAGÓGICA EN PLENO DESARROLLO
}

\author{
Economics of Education: an educational discipline \\ in high development
}

\author{
Pilar PINEDA HERRERO \\ Universitat Autònoma de Barcelona. Departament de Pedagogia Sistemàtica $i$ \\ Social. Edifici G-6.08193 Bellaterra. Barcelona.Correo-e: pilar.pineda@uab.es
}

Fecha de aceptación definitiva: marzo de 2001

BIBLID [(1130-3743) 12, 2000, 143-158]

RESUMEN

El artículo se centra en presentar la Economía de la Educación como disciplina pedagógica. Así, tras ubicar la disciplina en el conjunto de las Ciencias de la Educación y examinar las relaciones que establece con la Teoría de la Educación, se analizan los aspectos económicos de la educación, cuyo estudio da lugar a la aparición de la Economía de la Educación. A continuación se presenta su objeto de estudio y se analizan los conceptos implicados, para pasar a describir los orígenes y la evolución que la disciplina ha experimentado en sus breves 40 años de historia. Por último se presentan sus campos de estudio, profundizando en aquellos que presentan las líneas de investigación más punteras hoy, y apuntando las principales tendencias de desarrollo en el futuro.

Descriptores: Economía de la Educación, cuestiones económicas en educación, historia y desarrollo de la Economía de la Educación, áreas de investigación.

\section{SUMMARY}

This essay is focussed on the presentation of the Economics of Education as an educational discipline. The first part situates the discipline in the group of Educational 
Sciences and analyses its relations with the Theory of Education. The second part presents the Economics of Education's object and the different concepts linked with it. After that, the essay goes through the origins and the discipline's evolution in its short 40 years of history. The last part presents the study fields of Economics of Education, going deeper in these fields that are now the main research topics, and advancing the possible developments in the future.

Key words: Economics of Education, economics issues in education, history and developments of Economics of Education, research areas.

\section{La EConomía de la Educación en el Conjunto de las Ciencias de la Educación}

Antes de presentar la Economía de la Educación como disciplina y de profundizar en sus campos de estudio y desarrollos más recientes, es necesario determinar su carácter científico y ubicarla en el conjunto de las disciplinas pedagógicas. Ello entronca con el ya añejo debate relativo a la Pedagogía como ciencia o a la existencia de diversas Ciencias de la Educación. La postura más generalizada en la Pedagogía española en torno a este debate consiste en el reconocimiento de un conjunto de Ciencias de la Educación que estudian los fenómenos educativos, siendo la Pedagogía una de ellas, si cabe la principal, ya que sintetiza y da cohesión a la diversidad de perspectivas que analizan una misma realidad - la educación-, y normativiza sobre como debe ser la práctica de la misma. Así, la Pedagogía es una ciencia práctica, sintética y normativa que se ocupa de los diferentes procesos educativos formales, no formales e informales.

A pesar de este reconocimiento generalizado, en los últimos años se ha iniciado un debate sobre la Pedagogía y la Teoría de la Educación, centrado en demostrar el carácter sinónimo de ambas al referirse a la misma realidad, o las divergencias que las separan y que generan dos disciplinas diferentes.

Colom $(1992,1997)$ considera que el saber pedagógico lo proporcionan las llamadas Ciencias de la Educación, como conjunto múltiple y variado de disciplinas que estudian determinados aspectos de la educación y que inicialmente eran estudiados unitariamente por la Pedagogía. Entre este conjunto de disciplinas se halla la Teoría de la Educación, de procedencia anglosajona, que tiene por objeto el estudio teórico de la educación, por lo que se iguala a la concepción clásica de Pedagogía. Debido a su origen anglosajón, la Teoría de la Educación "rezuma toda la tradición pragmatista y utilitarista propia de estos paisajes" (Colom, 1992: 18), separándose así de la tradición pedagógica alemana, más reflexiva y filosófica.

Así, la Teoría de la Educación, dado su carácter pragmático, se ocupa de conocer la realidad educativa para intervenir en ella en vistas a su optimización. Para ello adopta e integra los conocimientos provenientes de las Ciencias de la Educación y de otras ciencias, y emite normas de acción que conduzcan a dicha mejora de la práctica educativa. 
Como puede observarse, el debate en torno a las Ciencias de la Educación permite empezar a ubicar la Economía de la Educación en su seno. Pero para proceder de lleno a dicha ubicación resulta aquí de gran utilidad distinguir entre los diversos saberes pedagógicos. Octavi Fullat (1983: 18) partiendo de la historia del pensamiento occidental sobre la educación, identifica tres tipos de reflexiones pedagógicas, a saber, reflexiones tecnológicas, reflexiones científicas y reflexiones filosóficas, cada una de las cuales conduce a una tipología de saber pedagógico.

De los tres tipos identificados nos interesa aquí especialmente la reflexión tecnológica; ésta proviene del vocablo griego téckne — saber hacer cosas, obrar con conocimiento de causa-, y se centra en identificar las técnicas pertinentes que conducirán a una práctica adecuada sobre los hechos educativos. Como indica Fullat, la técnica es el conjunto de procedimientos que conducen a realizar adecuadamente una tarea, y la técnica pedagógica es la aplicación de ese saber hacer, de esos procedimientos, a la realidad educativa. La reflexión tecnológica sobre la educación busca ese "saber hacer educado", y da lugar a las diferentes teorías, pedagogías y didácticas que integran el cuerpo de las Ciencias de la Educación.

Esta clasificación de las reflexiones pedagógicas y los análisis anteriores sobre las Ciencias de la Educación nos permite ubicar la Economía de la Educación como ciencia. La Economía de la Educación parte de una ciencia humana autónoma, la Economía, para abordar los numerosos aspectos e implicaciones económicas de los fenómenos educativos. Así, se sitúa en el grupo de las reflexiones científicas sobre la educación, ya que adopta los conocimientos empíricos de la economía y su metodología científica para analizar la complejidad de la educación en su conjunto. Como tal, es una Ciencia de la Educación independiente que analiza los fenómenos educativos desde la óptica económica y contribuye a su comprensión con las aportaciones que se desprenden de su enfoque disciplinar.

La Economía de la Educación, como Ciencia de la Educación, presenta un importante punto de unión con la Teoría de la Educación: la integración de conocimientos provenientes de otras ciencias y la emisión de normas de acción que conduzcan a la comprensión y optimización de la práctica educativa. Estos puntos de unión se verán con más detalle en los apartados posteriores, cuando se presenten los aspectos económicos de la educación y los campos de estudio de la disciplina que aquí nos ocupa.

Una vez situada la Economía de la Educación en el conjunto de las Ciencias de la Educación, nos hallamos en condiciones de presentar su objeto, su contenido, su origen y evolución, así como los campos de estudio trabajados hasta nuestros días. A ello se dedican los siguientes apartados.

\section{ASPECTOS ECONÓMICOS DE LOS PROCESOS EDUCATIVOS}

Entre el mundo económico y el mundo de la educación se dan numerosas relaciones e interacciones. De hecho, los procesos educativos integran, implican y 
determinan muchos aspectos económicos de gran importancia para su correcto funcionamiento. Son estos puntos de intersección entre la economía y la educación los que determinan el análisis económico de los procesos educativos, y serán analizados a continuación.

Es de aceptación universal considerar la educación como derecho humano básico. Incluso algunas personas van más allá y afirman que la educación, como derecho, debe ser gratuita para todos y que no caben consideraciones económicas en los debates sobre su organización. No obstante, hasta los derechos humanos básicos utilizan recursos escasos, y deben competir entre ellos en la repartición de las dotaciones presupuestarias. La sociedad ha de decidir cuánta educación desea consumir sin olvidar que con ello sacrifica la satisfacción de otras necesidades básicas (Johnes, 1993: 20). Por ello, las consideraciones económicas sobre la educación son importantes e ineludibles, ya que ésta absorbe gran cantidad de recursos productivos que podrían dedicarse a otras prioridades.

Paralelamente se observa que, una vez que los recursos productivos y económicos han sido destinados a la educación, pueden ser utilizados con mayor o menor eficacia. Del grado de eficacia y eficiencia con que se administren los recursos dependerá la rentabilidad de la educación, obtenida a partir del control económico de los costes y del cálculo de los beneficios que genera. La economía puede aportar a la Pedagogía sus modelos de gestión que garantizan una administración eficaz de los recursos económicos destinados a la educación, y que aumentan así su rentabilidad.

Desde los trabajos de Theodor Schultz y de Gary Becker a inicios de los años sesenta, la educación se considera una inversión en el futuro. La educación aumenta la productividad de la persona en el puesto de trabajo, por lo que puede contribuir a aumentar sus ingresos salariales. Esto conduce a una pregunta clave: ¿la educación genera un rendimiento mayor que otros tipos de inversión?, y ¿qué tipo de educación genera más rendimiento? La economía, con sus estudios sobre las tasas de rendimiento de diversas inversiones, puede aportar luz a estas incógnitas.

Por otro lado, si la educación es una inversión que aumenta los ingresos individuales, su efecto social es el aumento de los ingresos colectivos de la sociedad en su conjunto. Es decir, la educación genera también unos rendimientos sociales de tipo económico. Esto muestra otro punto de estrecha relación entre educación y economía: la contribución de la educación al crecimiento económico de las sociedades.

Los estudios sobre el rendimiento de la educación son fundamentales, ya que el rendimiento determina en gran medida la demanda de servicios educativos. La Administración educativa debe planificar los servicios de educación para que la oferta responda a las fluctuaciones de la demanda y también a las necesidades de cada territorio. Así, la planificación de la educación, función de decisiva importancia para la eficiencia de los servicios educativos, es otro ámbito de intersección entre los campos de la educación y de la economía.

Junto al tema de la eficacia de la educación aparece otro de gran relevancia hoy: la equidad de los sistemas educativos y las repercusiones que sobre la igualdad de 
oportunidades tienen las decisiones económicas en materia educativa. Es de todos aceptado que la educación aporta rendimientos privados y sociales, pero las cuestiones que fundamentan los estudios sobre la equidad son: ¿quién soporta realmente los costes de la educación?, ¿quién recibe de forma mayoritaria sus rendimientos? Así, el equilibrio entre la eficacia y la equidad de la educación es otro de los aspectos económicos de los procesos educativos.

Los servicios educativos se planifican, pero requieren de una financiación para su puesta en funcionamiento. Los sistemas de financiación de la educación pueden ser públicos, privados o mixtos en infinidad de combinaciones posibles. Los argumentos a favor y en contra de cada tipo de financiación tienen un marcado carácter ideológico, pero también económico - aunque la economía pueda tener mucho de ideológica-, por lo que encontramos otro punto de intersección que justifica el estudio económico de los fenómenos educativos.

Queda claro que los servicios educativos requieren de un sistema de financiación que les aporte los recursos necesarios para su funcionamiento. Pero estos recursos económicos deben ser gestionados y administrados de forma óptima. Para ello es necesario, recurriendo a los principios de la microeconomía, elaborar modelos y procedimientos de gestión económica de centros educativos que faciliten y optimicen la administración de los recursos económicos. Así, en la gestión económica de los centros educativos aparece otro punto de unión entre la economía y la educación.

Por último, se detectan fuertes interacciones entre el sistema educativo y los mercados laborales. De hecho, una de las finalidades de la educación es preparar a las personas para un puesto de trabajo, es decir, formar profesionales útiles para la sociedad. La situación actual de desempleo, de inestabilidad y precarización laboral, de cambios empresariales acelerados..., hace que las relaciones entre educación y mercado de trabajo sean complejas, y exige la colaboración de los agentes educativos y económicos para encontrar soluciones interdisciplinares a uno de los problemas clave de los últimos años: el trabajo como derecho básico.

Como se puede observar, las interrelaciones entre los mundos educativo y económico son muchas y todas ellas necesarias. Estos puntos de unión justifican el estudio económico de los fenómenos educativos y dan pie a la aparición de una disciplina nueva: la Economía de la Educación. Aunque estas interacciones son la base de los campos de estudio de la Economía de la Educación, varios de ellos, dado su marcado carácter aplicativo y optimizador, pueden considerarse también campos de estudio de la Teoría de la Educación; ello demuestra los puntos de unión entre ambas disciplinas que se apuntaban en el apartado anterior.

\section{CONCEPTO y OBjeto de la ECONOMía de la EdUCACión}

La Economía de la Educación es la disciplina que se ocupa del análisis de las implicaciones económicas de los procesos educativos. El énfasis se sitúa en la "economía" 
ya que es ésta la que aporta sus teorías y sus métodos para comprender los aspectos económicos de la educación. Si el acento se situase en la "educación", la disciplina se denominaría "Pedagogía Económica" o "Educación Económica", pero su objeto de estudio sería sustancialmente distinto, centrándose en los aspectos educativos que la educación aporta al hecho económico (Brunet-Valero, 1996).

De hecho, como afirman Brunet y Valero (1996: 16), en las relaciones que se han dado hasta hoy entre la economía y la educación, el rol primordial lo ha jugado la economía, siendo la educación la que ha cedido su terreno y no al contrario. La economía, como ciencia en expansión, fue ampliando su campo de estudio a otros ámbitos e introduciéndose en otros saberes científicos, creándose así disciplinas como la Economía de la Salud, la Economía de la Familia, la Economía de la Empresa y la Economía de la Educación entre otras.

No obstante, la Economía de la Educación es una más de las numerosas “ciencias auxiliares" — como se las denominaba antaño- que integran el rico y variado abanico de las Ciencias de la Educación. Como saber pedagógico, no normativiza cómo ha de hacerse la educación, sino que, a partir de los conocimientos empíricos de una ciencia externa a la Pedagogía como es la economía, describe unos aspectos concretos de los fenómenos educativos.

Este criterio es compartido por los economistas estudiosos de la educación, que consideran que la Economía de la Educación es una Ciencia de la Educación y a su vez una rama de la economía. Así lo ilustran Grao e Ipiña:

"Aunque por su objeto la Economía de la Educación puede ser considerada como una Ciencia de la Educación, en lo que hay que insistir, para dejar claro, es en que no se trata de una rama de la Pedagogía sino, como venimos recalcando, de la economía, con métodos, campos temáticos y desarrollos propios" (Grao e Ipiña, 1996: 22).

Llegados a este punto, se está en condiciones de dar una definición del término. Grao e Ipiña (1996: 22) definen la Economía de la Educación como la

"disciplina que estudia las leyes que regulan la producción, la distribución y el consumo de bienes y servicios educativos, es decir, de los productos propios de la actividad educativa, partiendo de la base que mientras las necesidades humanas son ilimitadas, los recursos son limitados, por lo que es preciso administrar esos recursos escasos".

Partiendo de esta definición, aquí se concibe la Economía de la Educación como la disciplina que estudia los aspectos económicos de la educación y los efectos que la educación tiene en la actividad económica a nivel de crecimiento y desarrollo.

La Economía de la Educación es una ciencia social económica aplicada y, como todo saber científico, fundamenta, describe y sistematiza su objeto formal: los aspectos y los efectos económicos de la educación. Como indican varios autores -Quintana (1980: 297), Grao e Ipiña (1996: 22) —, y a la luz de la concepción formulada arriba, el objeto de estudio de esta disciplina es doble: por un lado analiza el valor económico de la educación como factor de desarrollo económico, y 
por otro analiza los aspectos económicos de los procesos educativos, como los costes, la financiación, la rentabilidad y la planificación de la educación.

La consolidación de esta disciplina ha seguido un proceso interdisciplinar, en el sentido que se han hecho aportaciones importantes desde las diversas ciencias sociales. Así, aunque al principio las reflexiones provenían casi exclusivamente del campo de la economía, paulatinamente se han ido incorporando reflexiones provenientes de la sociología, el derecho, la psicología y fundamentalmente de la pedagogía, generándose de este modo una disciplina claramente interdisciplinar.

\section{Orígenes E historia de la ECONOMÍA DE LA EdUCACión}

\subsection{Desarrollo bistórico de la Economía de la Educación}

La educación ha estado siempre presente en las reflexiones de los economistas desde los inicios de la ciencia económica. No obstante, la Economía de la Educación, concebida bien como una rama especializada de la economía o como una Ciencia de la Educación, no vio la luz hasta finales de los años cincuenta. Se trata, por lo tanto de una disciplina joven, con apenas cuatro décadas de historia.

La Economía de la Educación como disciplina nace con la acuñación del término capital bumano por Theodore Schultz para referirse a la importancia que tiene la formación de las personas sobre la producción y el crecimiento económico. A partir de ese momento, la expresión capital bumano ha experimentado una difusión tal que actualmente es sinónimo de educación o formación (Grao e Ipiña, 1996). Es en torno a este concepto que otros autores como Becker, Mincer y el mismo Schultz fueron desarrollando la teoría a partir de la cual se creó un cuerpo doctrinal que se denominaría Economía de la Educación. En los casi cuarenta años de historia de la disciplina se han dado altibajos en su desarrollo y en su producción científica. A continuación se describe este proceso evolutivo, clasificado en etapas que coinciden aproximadamente con períodos de 10 años.

\subsubsection{Los años sesenta}

Esta década constituye la época de mayor apogeo y esplendor de la disciplina, hasta el punto que se la ha denominado los "años dorados" de la Economía de la Educación. Tras el anuncio por parte de Schultz en 1960 del nuevo término "capital humano" y del nacimiento de la teoría que lleva el mismo nombre, la disciplina empieza a desarrollarse a un ritmo frenético. El Journal of Political Economy dedica en 1962 un suplemento al tema Inversión en Seres Humanos, en el que aparecen los artículos pioneros de la disciplina, entre ellos los primeros capítulos de la obra de Gary Becker Human Capital (1964) que será la obra maestra de la disciplina.

El esplendor de esta década se debe en gran parte a la bonanza económica vigente y a la confianza absoluta en la educación como instrumento de movilidad 
y mejora social. Son los años de expansión del sistema educativo en los países occidentales, con un gasto social en educación de gran magnitud y en crecimiento constante.

Los ya citados Schultz, Becker, Denison y Vaizey, junto con Friedman, Kuznets, Mincer, Eicher, Bowen, Machlup, Harbison, Myers, Bowman, Anderson y muchos otros fueron desarrollando a lo largo de la década la nueva disciplina y creando el cuerpo doctrinal que daría origen a la Economía de la Educación (Grao e Ipiña, 1996: 17). Aunque con ciertas discrepancias, todos ellos concordaron en la importancia que la educación tiene para el desarrollo de las personas y de los pueblos, y dirigieron sus esfuerzos a identificar el grado de contribución de la educación al desarrollo y a crear modelos económicos para planificar la educación con eficacia, con lo que sentaron las bases para los estudios posteriores sobre economía y educación. Algunos de estos autores han visto reconocido su trabajo con el prestigioso Premio Nobel de Economía, como Friedman en 1976, Schultz en 1979 y Becker en 1992.

Varios organismos internacionales, como la OCDE, la Unesco y el Banco Mundial, han jugado un papel decisivo en el desarrollo de la disciplina en los años 60, patrocinando las reuniones de los especialistas y promoviendo algunos de los estudios más importantes sobre educación y desarrollo económico.

\subsubsection{Los años setenta}

Con la nueva década la sociedad occidental experimenta cambios importantes que afectarán al desarrollo de la Economía de la Educación como disciplina. Así, el aumento espectacular de la demanda de educación, que era la nota característica de la mayoría de los sistemas educativos desde el final de la Segunda Guerra Mundial, empieza a disminuir. Paralelamente, la confianza en la educación como instrumento de igualación social desaparece, para dar paso a un pesimismo sobre los efectos económicos de la educación y a una certeza sobre su contribución en el mantenimiento de las diferencias sociales.

Por otro lado, la mayoría de los gobiernos, preocupados por la inflación, el paro juvenil y el inminente exceso de personas con un elevado nivel de formación, empiezan a recortar los presupuestos educativos y a frenar la expansión de la educación. Así, las políticas educativas de los gobiernos en esta década se caracterizarán por el paso de la expansión cuantitativa a la reforma cualitativa de los sistemas educativos. El interés ya no está en garantizar que la educación sea accesible a toda la población sino que, una vez ya alcanzado ese objetivo y dados los cambios socioeconómicos del momento, el interés se centra en la mejora del sistema educativo y el uso eficaz de los recursos disponibles, siempre escasos.

Esta nueva realidad comporta cambios importantes para nuestra disciplina. En primer lugar, los economistas dejan de desempeñar el anterior rol fundamental en la Administración educativa, pasando a desempeñar este papel los psicólogos y los 
pedagogos como expertos en calidad de la educación. En segundo lugar, la producción científica en los temas de estudio iniciados en los años 60 disminuye considerablemente, y en algunos sectores llega a desaparecer. Ello es debido a la notable pérdida de interés en la contribución de la educación al crecimiento económico individual y colectivo.

Sin embargo, como indica Blaug (1996: 18), la Economía de la Educación no desaparece por completo como campo de estudio académico en esta década, sino todo lo contrario. En estos años se da un vigoroso desarrollo de la disciplina en nuevas direcciones, hasta tal punto que se habla de segunda generación de economistas de la educación para diferenciar los nuevos trabajos de los realizados en los años sesenta por los autores pioneros, que integran la primera generación.

Los autores de la segunda generación centran su interés en temas novedosos e inéditos hasta el momento, pero fundamentales para comprender las implicaciones económicas de la educación. Así, empiezan a estudiar la oferta educativa como factor determinante de la demanda y como elemento decisivo en toda planificación educativa. Se realizan estudios sobre la financiación de la educación, los diversos modelos existentes y sus consecuencias a nivel de eficacia y de equidad del sistema educativo. Otro ámbito de estudio novedoso en los años setenta son las relaciones entre la educación y el mercado del trabajo ya que, en un contexto donde el desempleo empieza a ser una característica constante, descubrir las posibilidades de la educación como herramienta de inserción laboral se convierte en una prioridad gubernamental y social.

Los cambios más importantes en la disciplina en estos años, y que recogen algunas de las líneas de estudio anunciadas, los protagonizan los economistas institucionalistas y los economistas radicales de Estados Unidos. Estos autores destacan la función socializadora de la educación en detrimento de la función profesionalizadora defendida por sus antecesores, con lo que invalidan parcialmente la versión pura de la teoría del capital humano. Estos importantes cambios en el cuerpo teórico de la Economía de la Educación se originan como críticas a la inicial teoría del capital humano en su versión pura y dan lugar a otras teorías alternativas y complementarias como la teoría del filtro (Arrow, 1973; Spence, 1973; Stiglitz, 1975), la teoría de las actitudes (Bowles, 1971; Gintis, 1974) y la teoría de la segmentación de los mercados de trabajo (Piore, 1971; Thurow, 1975).

\subsubsection{Años ochenta y noventa}

A inicios de los años ochenta la sociedad occidental experimenta de nuevo una serie de cambios socioeconómicos decisivos que afectarán de lleno a la Economía de la Educación. Este conjunto de cambios es la consecuencia de un fenómeno más global: la implantación del neoliberalismo económico a escala mundial. Ello, en el seno de una severa crisis financiera de alcance mundial, hace que se empiece a cuestionar el mantenimiento del Estado de bienestar y el papel del 
Estado en los temas económicos. Así, desde la posición hegemónica del neoliberalismo se replantea la relación entre educación y economía. (Casanova, 1994: 141).

Como indica Sante (1987: 39), el pensamiento neoliberal presente desde hace dos décadas sitúa

"la libertad de trabajo, de empresa y de consumo por encima de las eventuales ventajas de la economía "racionalizada" por la intervención del Estado. El protagonista de la vida pública no es el Estado, la colectividad, sino (...) el individuo, el consumidor".

Desde esta óptica, se plantea que el gasto público es excesivo e inadecuado, considerándolo como el causante de la inflación. Consecuentemente, los neoliberales proponen pasar del Estado de bienestar, iniciado en la postguerra y garantizador de unos servicios sociales para toda la población - educación, seguridad, sanidad, vivienda, etc.- a un "Estado mínimo" que promueva una amplia libertad en las actividades económicas de los individuos.

La aplicación del pensamiento neoliberal está generando cambios importantes en el ámbito de la educación y en su análisis económico. Así, el cuestionamiento de la eficacia del gasto social desde la nueva racionalidad económica impone reducciones en los recursos públicos destinados a educación. Este hecho va acompañado de un discurso de eficiencia, rentabilidad y productividad que impregna buena parte de los estudios sobre economía y educación.

Ya en los años noventa aparece un nuevo elemento que va a protagonizar buena parte de la investigación en Economía de la Educación: la evaluación. Así, se evalúan las instituciones educativas desde una óptica de eficacia, para verificar y controlar los procesos de gestión y los resultados alcanzados, con el ánimo de utilizar dichos resultados para determinar la financiación de la educación.

En definitiva, como indica Casanova (1994: 143), el escenario económico que rodea a la educación hoy se caracteriza por las crisis financieras mundiales, la hegemonía de los planteamientos neoliberales y la consecuente disminución del gasto público. En este contexto el análisis económico de la educación sigue siendo un ámbito de gran relevancia para el desarrollo de nuestra sociedad. Pero quizás convendría invertir la balanza y poner más énfasis en el polo "educación" que en el polo "economía".

Tras este repaso histórico de la evolución de la Economía de la Educación como disciplina podemos ver que, en un principio, la producción científica estuvo limitada casi exclusivamente a los países anglosajones, principalmente el Reino Unido y los Estados Unidos. Pero rápidamente se extendió a la Europa occidental, iniciando por Francia y siguiendo por el resto de países. Grao e Ipiña (1996: 17-18) elaboran la siguiente clasificación de los principales autores e investigadores que trabajan hoy en Economía de la Educación en los diferentes países:

- EE.UU.: Psacharopoulos y Woodhall desde el Banco Mundial; Levin y Carnoy desde la Universidad de Stanford; McMahon desde la Universidad de 
Illinois; Cohn en la Universidad de Carolina del Sur y Hoenack en la Universidad de Minnesota.

- Gran Bretaña: Blaug y Williams desde la London Economics School. Destacan también los trabajos de M. Kogan y colaboradores sobre Evaluación de Universidades o los de Sizer sobre indicadores.

- Francia: Eicher, Orivel, Jarousse y muchos más desde el IREDIJ (Instituto de Investigación sobre Economía de la Educación) de Dijon; Jallade y Neave desde el Instituto Europeo de Educación y Política Social; el equipo del CEREQ (Centro de Estudios y de Investigaciones sobre las Cualificaciones) o el del Instituto Internacional de Planificación de la Educación (IIPE) de la Unesco con Hallak como autor más destacado.

- Alemania: Destacan U. Teichler de la Universidad de Kessel y E. Frackman del HIs en Hanover.

- Países Bajos: Sobresalen el equipo del Cheps (Centro para Estudios sobre Política de Educación Superior) de la Universidad de Twente y el equipo de la Universidad de Amsterdam, donde se ubica Oosterbeek.

- Latinoamérica: Destaca especialmente Schiefelbein en el Centro de Investigación y Desarrollo de la Educación de Santiago de Chile.

También destaca el papel que varios organismos internacionales, como el Banco Mundial, la Unesco y la OCDE, han desempeñado en el desarrollo de la Economía de la Educación desde sus inicios hasta nuestros días, con la financiación y la realización de estudios y proyectos sobre diversos campos propios de la disciplina.

\subsection{La Economía de la Educación en España}

La historia de la Economía de la Educación en nuestro país se inicia en 1961, momento en que España subscribe un acuerdo con la OCDE para incorporarse al Proyecto Regional Mediterráneo. El proyecto, que vincula también a Portugal, Italia, Yugoslavia, Grecia y Turquía, tiene como objetivo el estudio de las necesidades educativas en el Mediterráneo hasta 1975. En su formulación se manifiesta claramente la estrecha relación existente entre educación y crecimiento económico y se indica la insuficiente preparación del factor humano español para el deseado desarrollo del país.

A partir de este primer trabajo en Economía de la Educación, la disciplina se ha desarrollado de forma desordenada y con muy pocos equipos consolidados que abriesen líneas de investigación fructíferas. La mayoría de los trabajos existentes se han realizado en la universidad, muchos por iniciativa propia de los autores, y algunos con financiación pública.

La Administración pública ha ido encargando algunos trabajos sobre el tema, como es el caso del MEC, el Consejo de Universidades y algunas administraciones autonómicas. La empresa privada ha demostrado muy poco interés por la Economía de la Educación, tanto en la financiación como en la producción de estudios. 
No obstante, se han realizado algunos trabajos patrocinados por El Corte Inglés, por el BBV, y por Argentaria entre otros ${ }^{1}$.

No obstante, en la última década la Economía de la Educación ha experimentado un desarrollo espectacular en nuestro país. Se identifican dos factores clave como impulsores de dicho desarrollo:

- La introducción en 1992 de la materia troncal "Economía de la Educación” en los nuevos planes de estudio de Pedagogía.

- La creación, también en 1992, de la Asociación de Economía de la Educación (AEDE) que reúne a estudiosos, investigadores y profesionales del tema, de la cual formo parte activa.

Este desarrollo ha dado como fruto la aparición de numerosos artículos y obras, tanto monográficos sobre un tema específico como generalistas en forma de manual. Así destacan autores como:

- Esteve Oroval, líder del equipo de investigación de la Universidad de Barcelona que es el más sólido de nuestro país, y primer catedrático de Economía de la Educación en España.

- Ginés Mora, vinculado a instituciones y equipos internacionales.

- José Luis Moreno, impulsor de la disciplina en el Archipiélago Canario.

- Jorge Calero, perteneciente al equipo de Oroval y autor de reconocidos estudios sobre los efectos del gasto público en educación.

- Julio Grao, autor e impulsor de las numerosas publicaciones del Gobierno vasco sobre temas de economía y educación.

Estos desarrollos recientes en nuestro contexto permiten predecir un futuro fructífero para la Economía de la Educación en España. Aunque, como se indicaba más arriba, los elementos del entorno imponen condiciones difíciles, hay que superarlas si lo que se pretende es contribuir a la mejora de la educación y, por extensión, de la sociedad en su conjunto.

\section{CAMPOS DE ESTUdio de la ECONOMÍA dE la EdUCACión}

En páginas anteriores se indicaba que la Economía de la Educación como disciplina tiene un doble objeto de estudio: estudia los efectos de la educación como factor de desarrollo económico, y analiza los aspectos económicos de los procesos educativos —costes, financiación, planificación de la educación, etc.- De este doble objeto de estudio se derivan sus campos de actuación, que son numerosos y han ido cambiando a lo largo del desarrollo histórico de la disciplina.

1. Para más información ver GraO e IPIÑa, 1996, p. 19. 
El punto de partida inicial, como ya se ha señalado, fue la teoría del capital humano que centró su interés en concebir la educación como una inversión en las personas, rompiendo con la tradición anterior que la consideraba un consumo tanto privado como público. Desde esta perspectiva se realizaron inicialmente tres tipos de trabajos:

- Estudios sobre las tasas de rendimiento de los diferentes niveles educativos, realizados en muchos países por iniciativa pública y privada.

- Estudios que valoran la contribución de la educación al crecimiento económico de un país, realizados inicialmente en países desarrollados y posteriormente en países en vías de desarrollo.

- Creación de métodos para racionalizar la toma de decisiones económicas en materia educativa y aportar así el criterio económico en la planificación de la educación. De los métodos propuestos destacan tres, ya citados anteriormente: el análisis de la demanda social, el enfoque de manpower planing y el análisis coste-beneficio.

Éstos son los campos de estudio básicos de los denominados economistas de la primera generación. En la segunda época, como ya se ha indicado antes, la Economía de la Educación experimenta un importante cambio de rumbo en relación a la línea marcada por sus antecesores, extendiendo considerablemente su campo de estudio a todos aquellos aspectos de la educación que tienen repercusiones económicas.

Una muestra de esta extensión de campo de estudio nos la da Woodhall (1985) al listar las principales áreas de investigación de la Economía de la Educación aparecidas hasta el momento y agruparlas en las siete siguientes:

1. Capital humano: en qué grado la educación aumenta la productividad del que la recibe.

2. Eficacia económica: cómo deben asignarse los recursos con el fin de producir bienes y servicios.

3. Contribución de la educación al crecimiento económico: en qué grado la educación contribuye al crecimiento económico.

4. Eficacia interna de la educación: qué relación existe entre los inputs y los outputs del proceso educativo.

5. Demanda de mano de obra educada: cómo prevenir o proyectar la mano de obra y con qué educación.

6. Financiación de la educación: quién y cómo debe pagar la educación.

7. Equidad y eficacia: cuál debe ser el punto de equilibrio entre la equidad y la eficacia del sistema educativo.

Desde la clasificación realizada por Woodhall han aparecido nuevos campos de actuación de la disciplina y se han reorganizado los existentes. Por ello se presenta aquí una clasificación alternativa que, si bien sigue las pautas básicas fijadas por Woodhall, introduce las nuevas perspectivas de estudio aparecidas recientemente. 
Así, los campos de estudio de la Economía de la Educación desde sus orígenes hasta nuestros días son:

- Aportaciones de las teorías económicas a la educación.

- Educación, crecimiento económico y desarrollo.

- Planificación de la educación.

- Costes y rendimiento de la educación.

- Financiación de la educación.

- Eficiencia y equidad en educación.

- Educación, ocupación y trabajo.

- Funcionamiento de los procesos educativos.

- Gestión económica de los centros educativos.

Actualmente, los campos de estudio que se están desarrollando con más vigor y que generan líneas de investigación punteras son especialmente dos:

- Eficiencia y equidad en educación:

Los estudios más recientes se centran en analizar los niveles de eficiencia y equidad de los sistemas educativos vigentes, especialmente en educación superior, y en proponer medidas y estrategias de financiación que contribuyan a la corrección de las disfunciones identificadas. Paralelamente se están desarrollado numerosos estudios sobre la evaluación del sistema educativo, tanto a nivel preuniversitario como universitario, convirtiéndose este tema en una de las líneas de investigación de más actualidad en Economía de la Educación.

- Educación, ocupación y trabajo:

El estudio de las relaciones entre educación y trabajo está adoptando una relevancia singular en Economía de la Educación, dados los cambios drásticos que acontecen en el mercado laboral y las repercusiones sociales que conllevan. Las últimas décadas se caracterizan por el fin de la sociedad industrial, que da paso a la sociedad del conocimiento y la información. Se crean nuevos entornos económicos globales y nuevas situaciones sociales que generan cambios importantes en el mercado de trabajo. Estos cambios se han convertido ya en las características del mercado laboral del nuevo milenio, que inevitablemente tienen graves consecuencias sociales, como el desempleo, la inestabilidad e inseguridad laboral, la polarización de la riqueza y la marginación de grandes grupos de población, entre otras.

Esta situación ha conducido a muchos economistas de la educación al estudio de las relaciones que se dan entre educación y trabajo para generar soluciones que mitiguen el apremiante problema del paro, que aumenten la empleabilidad de las personas, y que den más coherencia a las relaciones, a menudo contradictorias, entre el mercado laboral y el 
mundo de la educación. Las líneas de investigación aparecidas en este campo de estudio son numerosas y variadas, entre las que destacan:

- La adaptación de los sistemas educativos al mercado de trabajo.

- Los mecanismos de inserción profesional para desempleados.

- Las causas del desempleo de titulados.

- El fenómeno de sobreeducación o subocupación.

- La formación continua y en las organizaciones.

La mayoría de campos de estudio y de las líneas de investigación apuntadas como propias de la Economía de la Educación requieren un tratamiento interdisciplinar, con la intervención de disciplinas pedagógicas, económicas y sociológicas. Pero el ámbito científico que establece más nexos es el pedagógico, con la intervención de numerosas disciplinas como la Teoría de la Educación, la Pedagogía Social, la Pedagogía Laboral, la Educación Comparada, etc.

Sin embargo, como indican Grao e Ipiña (1996: 23), tanto los métodos como las herramientas analíticas y de tratamiento utilizados para investigar en estos campos han sido los propios de los economistas. Por lo tanto, la extensión del campo de estudio de la Economía de la Educación se ha realizado desde la óptica económica, ayudando así a comprender el fenómeno educativo en todas sus dimensiones, y posibilitando el abordaje pluridisciplinar del mismo.

Parece oportuno acabar esta descripción de la Economía de la Educación como disciplina con la siguiente afirmación de Esteve Oroval:

"Aunque el camino recorrido por esta disciplina es bastante fructífero, tanto en trabajos empíricos como en reflexiones teóricas, hoy por hoy no se puede afirmar que exista un cuerpo teórico sólido y ampliamente aceptado que explique el papel de la educación en el análisis económico de la sociedad.

Esto quiere decir que aún queda mucho trabajo por hacer y que se han de multiplicar los esfuerzos para ir avanzando en un campo tan básico para el conocimiento de la sociedad humana" (Oroval, 1994: 191).

Oroval indica claramente que queda mucho por hacer, y la existencia de una asignatura troncal en Economía de la Educación abre la puerta a la preparación de los profesionales de la Pedagogía para que desarrollen esta apasionante disciplina.

\section{BiBLIOGRAFÍA}

BeCKer, G. (1983): El capital humano. Alianza, Madrid.

Blaug, M. (1992): The Economic value of education. Edwar Elgar cop., Aldershot.

- (1996): "¿Dónde estamos actualmente en la economía de la educación?". En Oroval, E.

(ed.): Economia de la Educación. Ariel, Barcelona, pp. 17-37.

BRUNET, I. Y VAlero, L. F. (1996): Educación y economia. pPU, Barcelona. 
CALERO, J. (1998): Una evaluación de los quasimercados como instrumento para la reforma del sector público. Fundación BBv, Madrid.

Calero, J. y Bonal, X. (1999): Política educativa y gasto público educativo. Aspectos teóricos y una aplicación al caso español. Ediciones Pomares-Corredor, Barcelona.

CASANOVA, H. (1994): "Educació i Economia, una articulació complexa". Temps d'Educació, $11,137-145$.

CAstells, M. (1997): La era de la información. Economía, sociedad y cultura, vol. 1. Alianza Editorial, Madrid.

Castillejo, J. L. et al. (1994): Teoría de la Educación. Taurus, Madrid.

CoHn, E. y Johnes, G. (1994): Recent developments in the economics of education. Edwar Elgar cop., Aldershot.

Colom, A. (1992): "El saber de la Teoría de la Educación. Su ubicación conceptual». Teoría de la Educación. Revista Interuniversitaria, Iv, 11-19.

- (1997): Teorias e instituciones contemporáneas. Ariel, Barcelona.

Estefanía, J. (1998): Contra el pensamiento único. Taurus, Madrid.

Fullat, O. (1983): Filosofías de la educación. CEAc, Barcelona.

- (1999): Filosofía de la Educación. Síntesis, Barcelona.

GraO, J. e IpINAA, A. (1996): Economía de la Educación. Temas de estudio e investigación. Gobierno Vasco, Vitoria.

Instituto de Estudios Fiscales (1998): Educación y Economía. Monográfico en Hacienda Pública Española. Ministerio de Economía y Hacienda, Madrid.

JoHnes, G. (1993): The economics of education. St. Martin's Press, New York.

MEC (1999): Economia de la Educación. Monográfico de la Revista de Educación, 318, enero-abril. Madrid.

MARTIN-SHUMAN (1998): La trampa de la globalización. Taurus, Barcelona.

Moreno, J. L. (1998): Economía de la Educación. Pirámide, Madrid.

OCDE (1998): Human Capital Investment. An international comparison. OCDE, París.

Oroval, E. (1994): “Economia de l'educació. Una disciplina en formació". Temps d'Educació, 11, 191-194.

- (ed.) (1996): Economía de la Educación. Ariel, Barcelona.

- y Escardíbul, J. O. (1998): Economía de la Educación. Ed. Encuentro, Madrid.

Psacharopoulos, G. (1987): Economics of Education. Research and Studies. Pergamon Press, Oxford.

Quintana, J. M. (1980): Sociología de la educación. Ed. Hispano Europea, Barcelona.

SANTE, A. (1987): "Educación, libertad y eficiencia en el pensamiento y en los programas del neoliberalismom. Revista de Educación, 283.

Ventura, J. (1998): Perspectivas Económicas de la Educación. Ediciones Universidad de Barcelona, Barcelona.

VV.AA. (1995): "Economía de la Educación". Actas del v Congreso Nacional de Economía: "Economía de los servicios". Consejo de Colegios de Economistas, Las Palmas.

- (1997): Actas de las v Jornadas de la Asociación de la Economía de la Educación. Universidad de Castilla-La Mancha, Cuenca.

- (1998): vi Jornadas de la Asociación de la Economía de la Educación. Universidad de Cantabria y AEDE, Santander.

WoOdHALl, M. (1985): "Economics of Education". The International Encyclopedia of Education. Printe-Hall, New York. 